\title{
Design and Construction of a Sensor Analytics System for the Monitoring of the Parameters of a Plastic Injection Mould
}

\author{
Babalola Akinloluwa Samuel, Duncan Folley \\ School of Built Environment, Engineering and Computing, Leeds Beckett University, Leeds, United Kingdom
}

Email address:

akinloluwab@yahoo.com (B. A. Samuel)

To cite this article:

Babalola Akinloluwa Samuel, Duncan Folley. Design and Construction of a Sensor Analytics System for the Monitoring of the Parameters of a Plastic Injection Mould. International Journal of Sensors and Sensor Networks. Vol. 9, No. 1, 2021, pp. 25-29.

doi: $10.11648 /$ j.ijssn.20210901.14

Received: January 25, 2021; Accepted: February 2, 2021; Published: April 23, 2021

\begin{abstract}
Values of parameters such as temperature, humidity, number of plastic products and the location of plastic injection moulds are required to determine the efficiency of plastic injection moulds with a view to improving the quality of the outputs. This article determined the appropriate sensors for the measurement of these essential parameters in the most suitable form of representation of the data to aid a proficient analysis of the data. A network of sensors for the measurement and analysis of the parameters of plastic injection moulds in operation was designed and constructed. The outputs of these sensors were obtained by connecting the sensors to the General-Purpose Input/Output (GPIO) pins of a Raspberry Pi and writing a Python programme for the connected GPIO pins. The values of the outputs of these sensors were represented in graphical form. The connection of the Raspberry Pi and the sensors were done with a full-sized breadboard and jumper wires. A computer-aided design (CAD) of the connections was produced using Fritzing software. The appropriate sensors determined are MLX90614 infrared thermometer sensor, DHT11 humidity sensor, pixy2 vision sensor and Neo-6m GPS sensor. The output values of these sensors were plotted on a graph with the aid of a Python programme. The plastic industry has grown into a very broad industry as plastics are used to manufacture many materials. This makes it essential to ensure the efficiency of the industrial machines used in the making of these plastic products by using sensors to measure the parameters of a plastic injection mould in operation. This study therefore suggested the measurement of industrial plastic injection moulds with the use of the built sensors analytics system for the purpose of understanding and improving the performance of the injection mould.
\end{abstract}

Keywords: Injection Mould, Sensors, Parameters

\section{Introduction}

Injection moulding involves the transformation of plastic pellets into moulded plastic objects [24]. These moulded plastic objects take the sizes and shapes of the cavities of the plastic injection moulds. The materials used for plastic pellets are usually thermo plastics, thermo setting plastics and elastomers. Chen et al. defined injection moulding machine as a large, industrial machine used in the production of plastic objects [5]. Injection mould is a mechanical component of injection moulding machine. It consists of two parts which are the core side and the cavity side. The core side is movable while the cavity side of a plastic injection mould is fixed. The plastic pellets are transported to the fixed part of the injection mould while the movable part closes in on the fixed part for the moulding of the plastics. The movable part of the injection mould then opens and ejects the warm, moulded plastics after the operation of moulding. The ease in the moulding of plastics into different parts is due to the physical and chemical properties of plastics. Plastics are compressible under pressure and they shrink under colder temperature. Another property of plastics is its viscosity as they are usually less viscous at higher temperatures. The viscosity of plastics is essential in the production of quality 
moulded parts. The moulding process starts upon closure of the two sides of the injection mould which occurs after a hot, melted plastic has been fed into the mould under pressure. The melted plastic is then cooled after a period and after closure of the injection mould till it becomes stable and solidified. The moulded plastic part is ejected from the injection mould upon the reopening of the mould [7]. The quality of plastic products depends on the parameters of the injection moulding process such as the injection temperature and the cooling time [1].

Plastic products have become very common in this present age as they are frequently used as kitchen utensils, personal care products, toys and many more. Companies producing plastic injection moulds for companies producing plastic products need to remotely measure and monitor the performance of the plastic injection moulds in order to correct any faults or a decline in the performance of the injection mould. As the injection moulds are used in remote locations by companies producing plastic products, these companies do not have access to the parameters of the injection moulds in operation. The measurement and monitoring of the variables involved in the moulding processes of plastic objects which can be done by sensors are essential in optimizing the moulding processes [12]. Sensors can be defined as electronic devices used in the monitoring of parameters of a system without any form of distortion of the parameters [4]. There are various sensors designed for various purposes such as temperature sensors, proximity sensors, motion detectors and many more. According to Pacher et al., the construction of fast and reliable sensors is extremely vital in the controlling of the quality of moulded plastics [20].

\section{Literature Review}

John and Isaiah Hyatt, the brothers who fabricated and patented the injection moulding machine in 1872 might not have imagined the huge impact the invention would have on the world, especially in terms of infrastructural development and employment [9]. Injection moulding machines consist of hopper, drive unit, nozzle, clamping unit, extraction pin, injection mould, barrel, screw and hydraulic units [13]. The injection moulding process involves the filling of the hopper with plastic pellets and transporting it to the mould cavity under high temperature and pressure with the screw. The mould cavity (stationary part of the mould) then collects the melted plastic while the heaters are used to increase the temperature of the system which would melt the plastic pellets during the transfer of the pellets from the hopper to the injection mould. The core mould (movable part of the mould) then closes in on the cavity mould after the melted plastic has been injected. After the melted plastic is cooled and solidified, the core mould opens, and the extraction pin would then eject the moulded plastic part.

A new package programme (NPP) was developed to calculate various injection moulding parameters using the model of artificial neural networks (ANN) [19]. The ANN was used in obtaining optimum moulding parameters that facilitate minimum defects in moulded plastics such as injection time and volumetric shrinkage. The high performance of a plastic injection mould would facilitate quality plastic product to ensure the needs of customers and the safety requirements of plastic products are met. Tactile electronic measuring instrument and three-dimensional (3D) scanners are used to analyse shrinkages in moulded plastic parts [11].

Sensors can be used to obtain the necessary parameters of a plastic injection mould [2]. They are required to obtain the parameters of the injection moulds to determine and enhance the performance of a plastic injection mould. Sensors used in measuring the parameters of a plastic injection mould are embedded in the mould [2]. Artificial intelligence techniques such as artificial neural networks (ANN) and fuzzy logic approach are useful in the monitoring of the injection moulding process as they also allows for the modelling and forecasting of the moulding process [18].

The integration of sensors is progressing swiftly in the detection of imperfect input of robust control systems irrespective of its environment [16]. Sensor data analysis for equipment monitoring (SDAEM) is used to monitor whether a plant is operating within the specifications and to predict undesired events [8].

\section{Methodology}

\subsection{Appropriate Sensors for the Measurement of Parameters of a Plastic Injection Mould}

For effective measurement of the parameters of a plastic injection mould in operation, the following sensors were used for the prototype design of a sensors analytics system:

i. MLX90614 infrared thermometer sensor, for the measurement of temperature;

ii. Pixy2 vision sensor, for counting the number of plastic products;

iii. DHT 11 humidity sensor, for measuring humidity; and iv. Neo 6-m sensor, for geographical location.

Other relevant electronic components used for the design and construction of the sensor analytics system are the Raspberry Pi 4 Model B; full sized breadboard; male-tomale, male-to-female and female-to-female jumper wires; High-Definition Multimedia Interface (HDMI); cable keyboard; mouse and a screen.

\subsubsection{Temperature Sensors}

Temperature sensors are useful in various ways such as in the measurement and the regulation of the temperature of an electric iron and in controlling the performance of heaters in buildings especially during winter season. Temperature sensors are also used to ensure the quality of products and services are maintained or improved such as in the food industry. Certain foods and drinks are usually stored at certain temperatures to ensure they do not get spoilt during transportation and in shops before sales. Hospitals need to 
maintain certain temperatures for the effective storage of blood in blood banks and in rooms used for intensive care units (ICU) for the wellbeing of patients.

Temperature control is also very necessary in the operation of plastic injection moulds. Naik et al. stated that occurrences of defects in plastic injection mouldings predominantly depends on the temperature of the injection moulding machine [17]. This paper used MLX90614 infrared thermometer sensor for the measurement of injection mould temperature because of the high temperature range it can measure when compared with alternative temperature sensors such as Omron MEMS thermal infrared temperature sensor and TMP 006 temperature sensor having maximum temperature range of $50^{\circ} \mathrm{C}$ and $125^{\circ} \mathrm{C}$ respectively. MLX90614 infrared thermometer sensor is a type of temperature sensor that measures temperature of a mechanism within the range $-70^{\circ} \mathrm{C}$ to $380^{\circ} \mathrm{C}$ [15]. An electric iron was used in this study to test the performance of the MLX90614 infrared thermometer sensor as there was no access to a plastic injection mould for the testing.

\subsubsection{Vision Sensor}

A vision sensor is an electronic device that captures images and integrates it with the processing power of a computer to make decisions regarding to the position, quality, and the comprehensiveness of a manufactured product [3]. A vision sensor can recognize both stationary and moving objects by distinguishing the different patterns, characteristics, orientation and colours these various objects have. Vision sensors can be used in industries to observe the performance of machines and products in order to detect any defect such as in the alignment of machine in order to ensure that the standard of its products and services is maintained or improved. This paper used Pixy2 vision sensor to capture the moulded plastic products of the injection mould and to count them. Pixy2 camera is a small, fast, convenient, cheap electronic device that learns to detect object that it is taught [23].

\subsubsection{Humidity Sensor}

Humidity sensor is an electronic device that is used to measure the amount of water vapour in the surrounding environment. The DHT11 sensor was one of the components used in this study for the design of the sensors analytics system. DHT11 is a sensor that measures temperature and humidity and it consists of a calibrated digital signal output [6].

\subsubsection{Global Positioning System (GPS) Sensor}

GPS sensors are electronic devices used to detect the location of a system with the use of satellite-based navigation system [21]. Conventional GPS sensors obtain the position of an object by measuring the distances between its antenna and four or more satellites through the detection of the phase of the digital data sequence each satellite transmits [24]. Neo$6 \mathrm{~m}$ GPS sensor was one of the sensors used for the sensors analytics system in this study.

\subsection{Network of Sensors for the Measurement of the Parameters of a Plastic Injection Mould}

Each sensor was connected to the GPIO pins of the Raspberry Pi 4 Model B while Python programming language was used to obtain the outputs of the sensors. The Raspberry Pi 4 Model B+ consists of 40 pins of which pins 1 , 3, 5, 6 and 13 were connected to different ports of a full-sized breadboard with the use of male-to-female jumper wires. The circuit diagram of the connection of the pins of the Raspberry $\mathrm{Pi}$ and the sensors was produced using Fritzing software shown in Figure 1. Pins 1 and 6 of the Raspberry Pi are designed for $3 \mathrm{~V}$ power supply and Ground (GND) respectively while pins 3 and 5 are designed for Serial Data Line (SDA) and Serial Clock Line (SCL) respectively, with pin 13 designed for GPIO27 [10]. Pin 1 was used as Voltage common collector $(\mathrm{Vcc})$ for the DHT11 humidity sensor and the MLX90614 infrared temperature sensor. Pin 1 was connected to these sensors with the use of male to female jumper wires. The pins 3 and 5 of the Raspberry Pi were connected to the MLX90614 infrared thermometer sensor with male-to-female jumper wires and the breadboard ports. The pins were for the SDA and SCL connections respectively, and these pins are referred to as Inter-Integrated Circuit (I2C) pins. Pin 6 of the Raspberry Pi was connected to a port on the breadboard with the MLX90614 sensor and the DHT11 sensor connected to the same row of the pin 6 port with male-to-female jumper wires. Pins 13 of the Raspberry Pi were connected to the data output pins of the DHT11 sensor respectively with the use of the breadboard and male-to-female jumper wires. The Vcc, Ground (GND) and Transmitter (Tx) pins of the Neo-6m GPS sensor were connected to pins 2, 6 and 10 of the Raspberry Pi 4 Model B respectively.

The Raspberry Pi was programmed using Python language to obtain the values of the sensors every minute throughout the entire daily operation of the projects. However, the time interval could be changed depending on the requirement of customers which would occur after the testing of the sensor analytics system on a plastic injection mould.

\section{Results and Discussions}

The values of the temperature of the object measured by the MLX90614 thermometer sensor are plotted against the time of measurement as shown in Figure 2. The y-axis of the temperature graph labelled 'Object Temperature $\left({ }^{\circ} \mathrm{C}\right)$ ' contains the object temperature values while the x-axis of the graph, labelled 'Time' contains the time of measurement. The temperature of an electric iron plugged into a power source was obtained and plotted as shown in Figure 2.

The values of the humidity measured by the DHT11 humidity sensor are plotted against the time of measurement as shown in Figure 3. The y-axis of the humidity graph labelled 'humidity (\%)' contains the humidity values while the $\mathrm{x}$-axis, labelled 'time' contains the time of measurement. The name, the postcode and the latitude and longitude 
coordinates of the location is detected by the Neo-6m GPS sensor as shown in Figure 4.

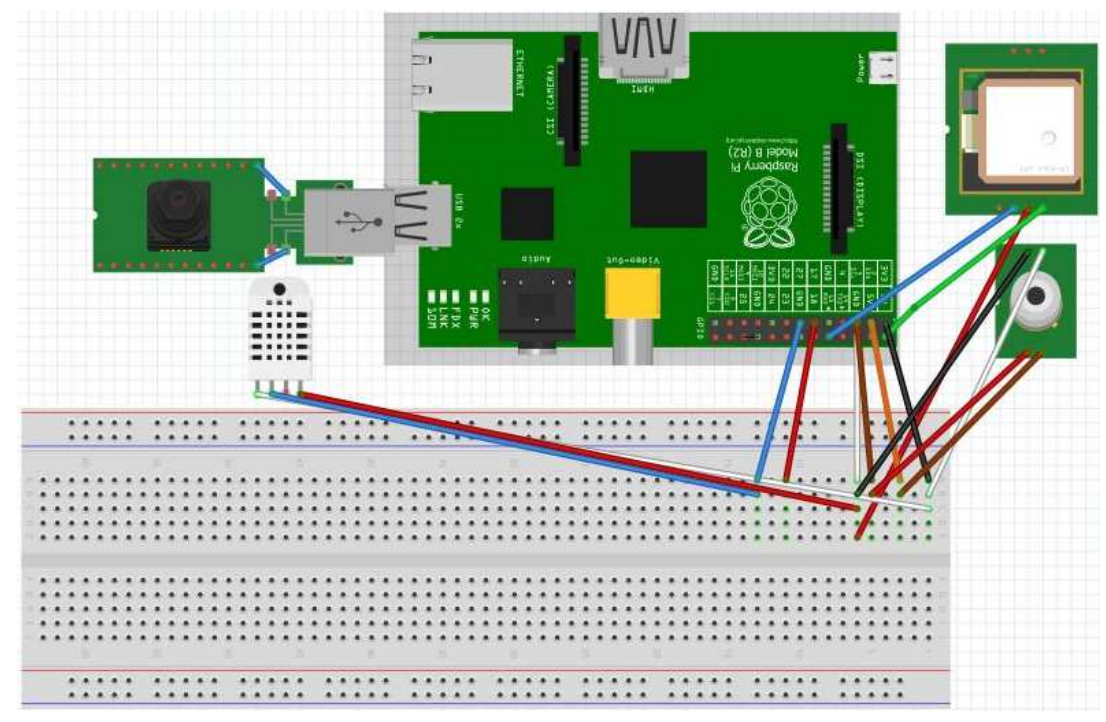

Figure 1. Circuit-aided design (CAD) of connection of components.

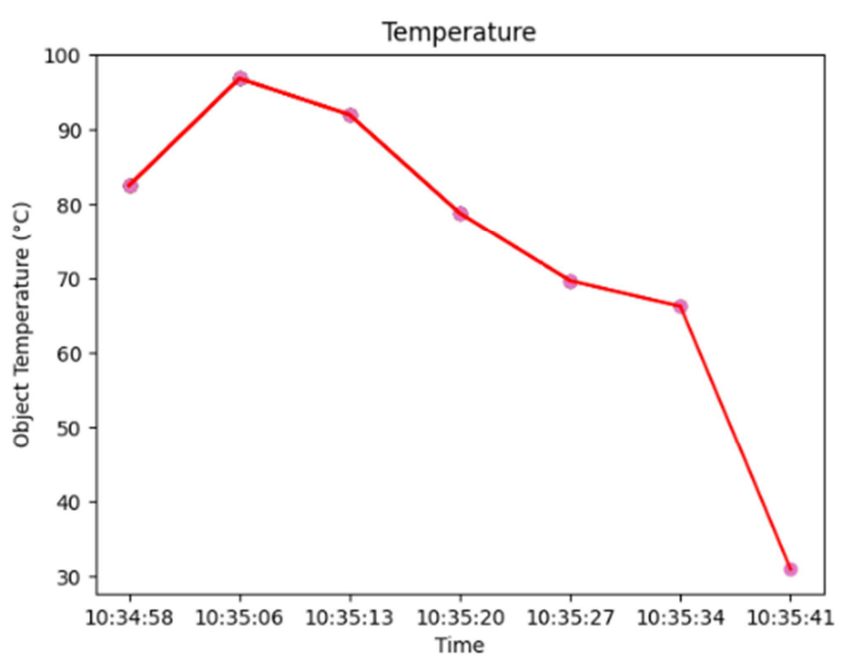

Figure 2. Object Temperature Graph.

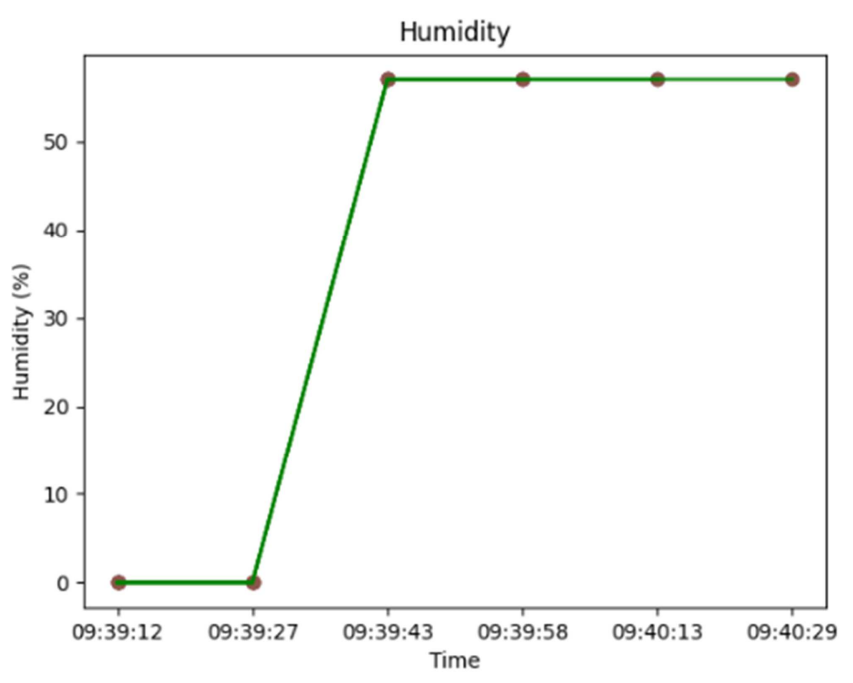

Figure 3. Humidity Graph.

\section{〉> \&Run unilever,py \\ Lat itude $=53.8135725$ and Longitude $=-1.5840338333333333$ Back Stanmore Street, Burley, Leeds, West Yorkshire, Yorkshire and the Humber, England, LS4 2RU, United Kingdom Amhinnt tammanont...men 10ar.}

Figure 4. Geographical Location.

The collection and visualization of correlated data obtained from sensors enable easy interpretation of data by human beings [14]. The visualization of the data obtained from the sensors used in this study enables easy evaluation of these data to understand the performance of a plastic injection mould. As it is essential to monitor the injection moulding process [22], the sensor analytics system allows for easy monitoring of the injection moulding process. This is to ensure high quality of plastic product are moulded by the plastic injection moulds [18].

\section{Conclusion}

The plastic industry has become very broad as plastics are used to manufacture many materials for human usage. Many homecare products, electronic equipment, medical equipment, kitchen utensils and many more are made from plastics. It has therefore become more important to ensure the efficiency of the industrial machines used in the making of these plastic products for safety and customer's satisfaction.

This study focused on the design and construction of a sensor analytics system with a view to monitoring the essential parameters of a plastic injection mould. The appropriate sensors for the measurement of these parameters were chosen from the numerous sensors available for different purposes. The sensor analytics system was constructed by integrating these sensors to the Raspberry Pi 
with the use of breadboards, jumper wires and USB cable. The sensors used were selected based on the specifications in comparison to the conventional parameters of a plastic injection mould in operation. The sensor analytic system developed measured ambient and object temperature, geographical location, number of objects, and humidity. It can therefore be concluded that the appropriate sensors for the sensing and measurement of the necessary parameters of a plastic injection mould in operation are MLX90614 infrared thermometer sensor, Neo-6m GPS sensor, DHT11 humidity sensor and the Pixy2 vision sensor.

This study proposed that the sensors analytics system be applied on an industrial plastic injection mould to measure and display the various parameters of the injection moulds for the purpose of understanding and improving the performance of the injection mould.

\section{References}

[1] Abohashima, H., Aly, M., Mohib, A., Attia. H. (2015) 'Minimization of Defects Percentage in Injection Molding Process using Design of Experiment and Taguchi Approach', Industrial Engineering \& Management, 4 (179). doi: 10.4172/2169-0316.1000179.

[2] Ageyeva, T., Horváth, S. and Kovács, J. G. (2019) 'In-Mold Sensors for Injection Molding: On the Way to Industry 4.0', Sensors (Switzerland), 19 (16). doi: 10.3390/s19163551.

[3] Automation, H. (2018) 'The Case for Automation with Machine Vision', HTE Automation.

[4] Chaurasiya, H. (2012) 'Recent Trends of Measurement and Development of Vibration Sensors', 9 (4), pp. 353-358.

[5] Chen, B., Wu, H., Zhou, H., Sun, D. (2020) 'EMP: Extended kalman filter based self-adaptive mold protection method on a toggle mechanism', Applied Sciences (Switzerland), 10 (3). doi: 10.3390/app10030940.

[6] D-robotics (2010) 'Temperature Sensor DHT 11 Humidity \& Temperature Sensor', D-Robotics, p. 9. Available at: www.droboticsonline.com.

[7] Dangel, R. (2016) Injection Molds for Beginners, Injection Molds for Beginners. Edited by M. Smith. Hanser Publishers. doi: 10.3139/9781569908198.fm.

[8] Garcia, A., Bentes, C., de Melo, R., Zadrozny, B., Penna, T. (2010) 'Sensor data analysis for equipment monitoring', Knowledge and Information Systems, 28 (2), pp. 333-364. doi: 10.1007/s10115-010-0365-1.

[9] Goodship, V. (2004) Practical guide to injection moulding, Metal Powder Report. doi: 10.1016/s0026-0657(97)91031-6.

[10] Hawkins, M. (2019) Simple Guide to the Raspberry Pi GPIO Header - Raspberry Pi Spy. Available at: https://www.raspberrypi-spy.co.uk/2012/06/simple-guide-tothe-rpi-gpio-header-and-pins/ (Accessed: 30 August 2020).

[11] Jozwik, J., Tofil, A. and Lukaszewicz, A. (2019) 'Application of modern measurement techniques for analysis of injection moulding shrinkage', in Engineering for Rural Development, pp. 1742-1748. doi: 10.22616/ERDev2019.18.N294.
[12] Karbasi, H. and Reiser, H. (2006) 'Smart Mold : Real-Time In-Cavity Data Acquisition', First Annual Technical Showcase \& Third Annual Workshop.

[13] Kumar, A. (2019) Injection Molding: Definition, Parts, Process, Advantages, Disadvantages, and Defects. Available at: https://learnmechanical.com/injection-molding-processdefects-parts/ (Accessed: 25 January 2021).

[14] Logre, I., Mosser, S., Collet, P., Riveill, M. (2014) 'Sensor Data Visualisation: A Composition-Based Approach to Support Domain Variability', in European Conference on Modelling Foundations and Applications, pp. 101-116. doi: 10.1007/978-3-319-09195-2 7.

[15] Melexis (2019) Digital Non-Contact Infrared Thermometer (MLX90614), Melexis. Available at: https://www.melexis.com/en/product/mlx90614/digital-plugplay-infrared-thermometer-to-can.

[16] Morales-Herrera, R., Fernandez-Caballero, A., Somolinos, A., Sira-Ramirez, H. (2017) 'Integration of Sensors in Control and Automation Systems', Journal of Sensors, 2017. doi: $10.1155 / 2017 / 6415876$.

[17] Naik, M., Shetty, P., Kotresh, K., Avinash, L. (2019) 'Prevention of defects in injection molding process in the manufacturing of ballpoint pen', International Journal of Recent Technology and Engineering, 8 (3), pp. 4932-4937. doi: 10.35940/ijrte.C5590.098319.

[18] Ogorodnyk, O. and Martinsen, K. (2018) 'Monitoring and Control for Thermoplastics Injection Molding A Review', in 11th CIRP Conference on Intelligent Computation in Manufacturing Engineering. The Author(s), pp. 380-385. doi: 10.1016/j.procir.2017.12.229.

[19] Özek, C. and Çelk, Y. H. (2012) 'Calculating Molding Parameters in Plastic Injection Molds with ANN and Developing Software', Materials and Manufacturing Processes, 27 (2), pp. 160-168. doi: $10.1080 / 10426914.2011 .560224$.

[20] Pacher, G., Berger, G., Friesenbichler, W., Gruber, D., Macher, J. (2014) In-mold sensor concept to calculate process-specific rheological properties. AIP Conference Proceedings, American Institute of Physics, pp. 179-182.

[21] Scientific, C. (2020) Global Positioning System (GPS) Sensors: Receivers with antennas. Available at: https://www.campbellsci.eu/gps (Accessed: 14 September 2020).

[22] Speight, R., Coates, P., Hull, J., Peters, C. (1997) 'In-line process monitoring for injection moulding control', in Proceedings of the Institution of Mechanical Engineers, Part E: Journal of Process Mechanical Engineering, pp. 115-128. doi: 10.1243/0954408971529601.

[23] Studio, S. (2018) Pixy CMUcam5 Smart Vision Sensor. Available at: https://www.amazon.com/Pixy-CMUcam5Smart-Vision-Sensor/dp/B00IUYUA80.

[24] Teague, E., How, J., Lawson, L., Parkinson, B. (2013) 'GPS as a Structural Deformation Sensor', AIAA Guidance, Navigation, and Control Conference (GNC), pp. 787-795. doi: 10.2514/6.1995-3258.

[25] Thyregod, P. (2001) Modelling and Monitoring in Injection Molding, Thesis page 155. doi: 10.14075/j.jgg.2017.06.015. 